\title{
Cenário da publicação científica sobre a Indústria 4.0 no Brasil: Uma revisão bibliométrica
}

\author{
A scenario of the scientific publication on Industry 4.0 in Brazil: A bibliometric review \\ Escenario de publicación científica sobre Industria 4.0 en Brasil: Una revisión bibliométrica
}

Recebido: 12/03/2021 | Revisado: 20/03/2021 | Aceito: 16/04/2021 | Publicado: 29/04/2021

\author{
André Henrique Ivale \\ ORCID: https://orcid.org/0000-0002-4901-1195 \\ Universidade Paulista, Brasil \\ E-mail: andreivale_bt@hotmail.com \\ Mário César da Silva \\ ORCID: https://orcid.org/0000-0003-0417-4908 \\ Universidade Paulista, Brasil \\ E-mail: mario.silva@ docente.unip.br \\ Irenilza de Alencar Nääs \\ ORCID: https://orcid.org/0000-0003-0663-9377 \\ Universidade Paulista, Brasil \\ E-mail: irenilza.naas@docente.unip.br
}

\begin{abstract}
Resumo
O objetivo do presente estudo é apresentar a implementação da indústria 4.0 no Brasil. Foi realizado o levantamento bibliográfico dos artigos científicos e publicações das instituições que estudam os modelos tecnológicos e industriais. Os artigos tiveram como critério de avaliação e inclusão a data de publicação (2012-2019) e o idioma (português), com foca nas áreas de Engenharia de Produção. A análise dos artigos indica que as pesquisas brasileiras apontam como base desta nova indústria a internet das coisas, sistemas ciber-físicos, automação, big data e inteligência artificial como temas importantes. O presente estudo ponta que o modelo da indústria 4.0 é o novo a nova tendência para atender a um mercado cada vez mais exigente, flexível e fortemente voltado para a era digital. A formação do Engenheiro de Produção deve atender também a estas demandas.
\end{abstract}

Palavras-chave: Revolução industrial; Revolução 4.0; Indústria 4.0 no Brasil.

\begin{abstract}
The objective of the present study is to present the implementation of industry 4.0 in Brazil. A bibliographic survey of scientific articles and publications of institutions that study technological and industrial models was carried out to this end. The articles had as their evaluation and inclusion criteria the date of publication (2012-2019) and the language (Portuguese), focusing on the areas of Production Engineering. The articles' analysis indicates that Brazilian research points to the basis of this new industry, the internet of things, cyber-physical systems, automation, big data, and artificial intelligence as essential themes. The present study points out that the industry 4.0 model is the new and the new trend to meet an increasingly demanding, flexible, and strongly focused market for the digital age. The training of the Production Engineer must also meet these demands.
\end{abstract}

Keywords: Industrial revolution; Revolution 4.0; Industry 4.0 in Brazil.

\section{Resumen}

El objetivo del presente estudio es presentar la implementación de la industria 4.0 en Brasil. Se realizó un relevamiento bibliográfico de artículos científicos y publicaciones de instituciones que estudian modelos tecnológicos e industriales. Los artículos tuvieron como criterios de evaluación e inclusión la fecha de publicación (2012-2019) y el idioma (portugués), con un enfoque en las áreas de Ingeniería de Producción. El análisis de los artículos indica que la investigación brasileña apunta a las bases de esta nueva industria, el internet de las cosas, los sistemas ciberfísicos, la automatización, el big data y la inteligencia artificial como temas importantes. El presente estudio señala que el modelo Industria 4.0 es la nueva y la nueva tendencia para hacer frente a un mercado cada vez más exigente, flexible y fuertemente enfocado a la era digital. La formación del ingeniero de producción también debe satisfacer estas demandas.

Palabras clave: Revolución industrial; Revolution 4.0; Industria 4.0 en Brasil.

\section{Introdução}

No século XXI, no ano de 2011, surgiu um projeto do Governo Federal da Alemanha, o High-Tech Strategy 2020, 
com a iniciativa estratégica de alcançar o reposicionamento frente a tecnologias de ponta e competitividade para as suas indústrias (Kagermann et al., 2013), fortalecendo assim a indústria manufatureira e introduzindo o conceito da indústria 4.0 ou quarta revolução industrial. Com o objetivo estratégico de explorar o potencial econômico e de inovação resultante do impacto das tecnologias de informação e comunicação na indústria, o principal foco da Indústria 4.0 é melhorar as cadeias de valor em todas as fases do ciclo de vida do produto (Firjan, 2016), revolucionando as linhas de montagem e gerando produtos inovadores e customizados (Siemens, 2016).

As três revoluções anteriores trouxeram um ganho de escala e produtividade de produção e a $4^{a}$ revolução industrial está em curso e trará também mudanças com novas perspectivas para a indústria, sendo caracterizada pela maior integração da Internet nos processos produtivos, sensores menores e mais baratos e inteligência artificial aplicada nas máquinas, tornando-as tomadoras de decisões de seus próprios processos de forma autônoma e inteligente (Lee et al., 2014). Isto permitirá avanços socioeconômicos com a integração da economia global, criando novas demandas para serviços e produtos. A quarta revolução industrial tem o marco de novas tecnologias que estão se fundindo com os mundos físico, digital e biológico, e está impactando a economia com a forma de consumismo e relacionamentos com os produtos. Mas o que realmente a diferenciou de todas as outras foi a capacidade de gerar velocidade, amplitude e profundidade (ABDI, 2019). A indústria 4.0 propõe todas essas mudanças através de alguns pilares de tecnologia: A conexão entre equipamentos, processos e softwares, através de sensores; Cyber sistemas com máquinas com capacidade de memória; Armazenamento de informações em servidores virtuais (cloud); Comunidades com interconexões e informações compartilhadas; Conteúdo com a garantia de qualidade de informação; e Customização que permita a manufatura ajustável, flexível e prática (Falcão, 2019).

O tema, mesmo que em estágio inicial, contém publicações acadêmicas e artigos científicos disponíveis nas bases consultadas. O presente estudo procura, através de uma revisão bibliométrica, relacionar as principais abordagens sobre a indústria 4.0, na perspectiva da Engenharia da Produção e a tendência das tecnologias em território brasileiro. Este estudo dispõe a aplicabilidade dos conceitos da Indústria 4.0 nas áreas/subáreas na perspectiva da Engenharia de Produção e a tendência das tecnologias em território nacional.

\section{Referencial Teórico}

\subsection{Revoluções Industriais}

Historicamente a indústria foi um fator dominante para a aceleração do crescimento econômico, originando assim as revoluções industriais juntamente com os avanços tecnológicos. Todo processo de evolução de meios de comunicação, industrialização e tecnologia começou com o evento conhecido como a primeira revolução industrial, que surgiu na Inglaterra no fim do século XVIII, onde a ciência descobriu a utilidade do carvão como fonte de energia e então foi inventado e depois aperfeiçoado por James Watt as máquinas a vapor (Venturelli, 2017). Vista a possibilidade de inovação, através dos anos, novos métodos de produção que pudessem ampliar o lucro dos capitalistas foram sendo desenvolvidos (Sousa, 2019). A segunda revolução industrial ocorreu em meados do século XIX e nessa nova etapa o uso da energia elétrica e os combustíveis fósseis permitiu o desenvolvimento de novos mercados.

A partir das últimas décadas do século XX, uma nova etapa veio consolidando a terceira revolução industrial, também conhecida como revolução informacional, que se trata da introdução dos recursos tecnológicos da informática no processo produtivo e a globalização de informações com fácil acesso (Boligian \& Alves, 2010). As últimas três revoluções industriais foram marcadas pelo avanço tecnológico que acompanhou o desenvolvimento econômico e social da humanidade. A quarta revolução industrial, atualmente em curso, traz desafios de alta relevância para o Brasil, importantes impactos tecnológicos em diversos setores manufatureiros e aplicação transversal. 


\subsection{Indústria 4.0 e suas Tecnologias}

O termo Indústria 4.0 surgiu em 2011 na Alemanha, o governo alemão em uma parceria com universidades, empresas e centros tecnológicos, propôs incentivo e patrocínio para o investimento em um novo modelo industrial. Toda essa mudança está relacionada com a descentralização do controle de processos e os avanços da tecnologia de dispositivos inteligentes interconectados, em toda a cadeia de produção e de logística (Aevo, 2017). A quarta revolução industrial tem o marco de novas tecnologias que estão se fundindo com os mundos físico, digital e biológico, e está impactando a economia global.

A indústria 4.0 propõem mudanças através de alguns pilares de tecnologia como, a conexão entre equipamentos, processos e softwares, através de sensores; cyber sistemas com máquinas com capacidade de memória; armazenamento de informações em servidores virtuais (cloud computing); comunidades com interconexões e informações compartilhadas; conteúdo com a garantia de qualidade de informação; e, customização que permita a manufatura ajustável, flexível e prática.

O uso de Big Data possibilita organização e análise de dados em grandes quantidades e de diversas fontes e se tornou uma das principais ferramentas da indústria 4.0. Realizando a junção do mundo físico com o mundo digital, processando uma grande quantidade de dados e informações e gerando hiper conectividade (Taurion \& Cezar, 2013). Aplica-se o uso da robótica na indústria em diversas áreas, para o aumento da eficiência e produtividade, na doméstica (conectividade e conforto), nas áreas urbanas (transportes e planejamento) e na área militar (melhorias nas estratégias táticas e vantagens de armamento) (Wolf et al., 2009).

A Internet das Coisas - IOT disponibiliza conectividade entre tecnologias através de sensores e dispositivos que geram dados que são analisados (data analytics) e assim permitem a tomada de decisão em tempo real. Que é o avanço no uso de objetos e equipamentos que já eram utilizados antes da indústria 4.0 e que com a junção da IOT e a conectividade tornaram os processos mais eficientes (Santos et al., 2016). A computação em nuvem (cloud computing) dispõe aumento na velocidade e capacidade nos processamentos, alta quantidade de dados passíveis de integração e economia de hardware, além da alta segurança no armazenamento de dados, facilitando o acesso de dados e serviços independentemente da localização (Souza et al., 2017).

\subsection{A Indústria 4.0 no Brasil}

A indústria representa hoje menos que 10\% do PIB e segundo Sakurai (2018) o Brasil com relação aos avanços tecnológicos, se encontra em atraso, e isso torna-se ainda mais evidente e concreto quando comparado com outros países. Dados mostram que o Brasil ganhou cinco posições no índice Global de Inovações (Gii, 2018) subindo de $69^{\circ}$ para o $64^{\circ}$ lugar de 126 países em um ranking das economias com maiores capacidades e sucesso de inovação. Entre as áreas em que o país se destacou estão gastos com P\&D, importações e exportações líquidas de alta tecnologia, publicações científicas. No entanto, dados do Ministério da Ciência, Tecnologia, Inovações e Comunicações (MCTIC, 2018) mostram que gastos em Pesquisa e Desenvolvimento (P\&D) ainda são baixos (US\$43 bilhões - 2,5\% do PIB) quando equiparado a outros países como China (US $\$ 407$ bilhões - 2,9\% do PIB) e Estados Unidos (US $\$ 496$ bilhões - 2,5\% do PIB), por mais que em relação ao seus respectivos PIB, indica que o Brasil está investindo na mesma proporção. Não se deve ter pensamentos igualitários, quando se sabe que os dois primeiros países são os que têm maior potencial econômico, sendo classificados em primeiro e segundo lugar

Uma pesquisa da Confederação Nacional da Indústria (CNI, 2016) sobre a digitalização mostra que apenas $48 \%$ (menos da metade) da indústria nacional utiliza tecnologias digitais e raramente se aproveita o potencial destas para promover mudanças disruptivas, como desenvolver novos produtos ou novos modelos de negócios. O principal desafio do país apontado pela pesquisa é aproximar especialistas e indústrias para a ampliação de conhecimento sobre os benefícios que essa revolução pode trazer para a cadeia produtiva, os altos custos de implantação e a baixa qualificação da mão de obra são, entretanto, fatores limitantes (Vermulm, 2018). 
As indústrias precisam tomar algumas ações para se adaptar a essa nova era. Devem enxugar seus processos produtivos consagrando a manufatura enxuta, adotar eficiência energética e produção mais limpa. Devem também requalificar seus funcionários para que estejam aptos e tenham conhecimento destas novas tecnologias digitais. A inserção de tecnologias disponíveis e de baixo custo será primordial para a introdução da indústria 4.0, como por exemplo o uso da inteligência artificial que permite agir com antecedência, antevendo problemas como quebras de equipamentos e planejar manutenções preditivas. E por fim as indústrias brasileiras deveriam ousar no desenvolvimento de produtos inteligentes e conectados, que utilizem tecnologias capazes de afetar o mercado internacional, investindo em pesquisas, desenvolvimento e inovações (Firjan SENAI, 2019).

\section{Metodologia}

Trata-se de uma pesquisa bibliográfica, utilizando o processo indutivo para a análise de dados e informações, levantados de dados bibliográficos (Pereira et al., 2018). Para definição das etapas, utilizamos como base a revisão bibliométrica integrativa (Figura 1), objetivando a sumarização dos trabalhos já realizados, visando à conclusão geral do presente estudo (Broome, 2006; Beyea \& Nicoll, 1998).

Figura 1 - Etapas da revisão bibliométrica e integrativa.

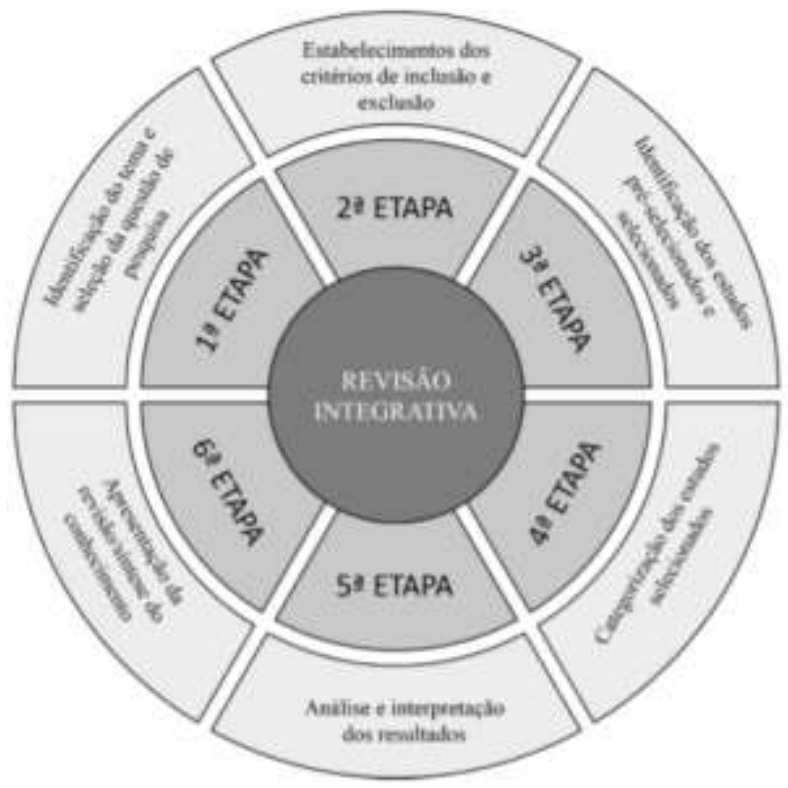

Fonte: Adaptado de Botelho et al. (2011)

A escolha do método de revisão, originalizou-se mediante ao tema não estar em seu estado primitivo da arte, visando à contribuição para o desenvolvimento teórico e sua aplicabilidade prática direta (Whittemore \& Knalf, 2005). A Figura 1 descreve as etapas (seis no total) e, em cada etapa, a tarefa a ser empreendida.

\subsection{Levantamento dos dados}

Após seleção e definição da pesquisa, o segundo passo foi realizar um levantamento e estudo de todos os artigos publicados, entre o período de 2012 à 2019, a fim de verificar a difusão do tema e quais áreas do conhecimento estavam sendo abordadas, classificar e filtrar os dados de acordo com os critérios de exclusão adotados e comparar os resultados obtidos com o que a Associação Brasileira de Engenharia de Produção (ABEPRO, 2019b) define como conhecimento e áreas da Engenharia 
de Produção no Brasil frente a Indústria 4.0, para obter uma perspectiva do tema e sua evolução, bem como a sua perspectiva futura. Através de pesquisa nas bases de dados da SCIELO, Google Acadêmico e ABEPRO, foi possível realizar o levantamento dos artigos. Foram selecionados todos os trabalhos publicados pela sociedade científica brasileira, entre o período citado, com foco na engenharia de produção para um posterior tratamento e análise dos mesmos.

Após o levantamento, foram selecionados os artigos coerentes com o tema a ser estudados e catalogados para um primeiro tratamento. Os artigos publicados entre o período selecionado, passaram por um filtro onde alguns critérios foram estabelecidos, para seleção ou exclusão do mesmo para uma segunda etapa (Figura 2). Entre esses critérios, podemos citar: Tema e assunto do estudo, fonte de pesquisa e publicação, relevância e citações e artigos referente à área de conhecimento da engenharia de produção.

Figura 2 - Filtragem e tratamento dos dados analisados na revisão bibliométrica.

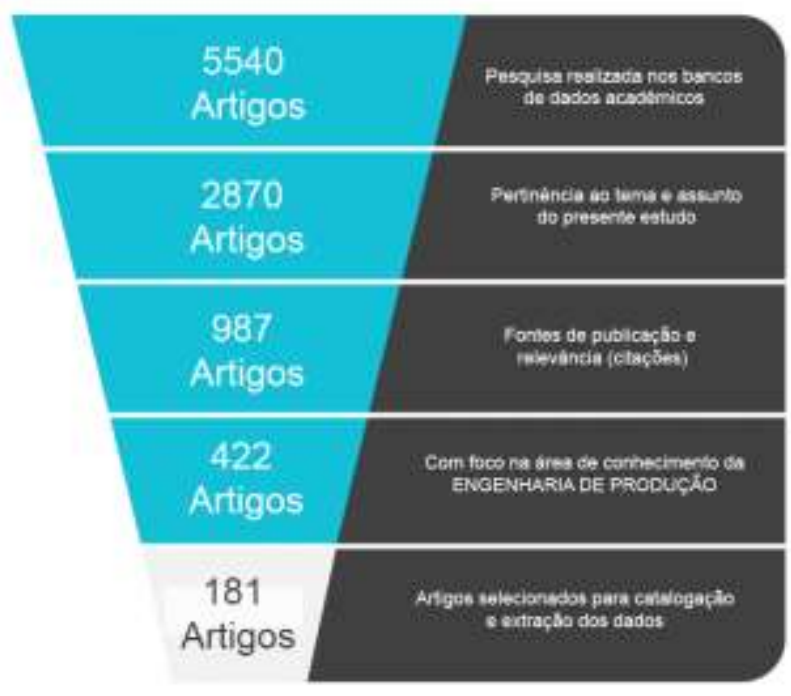

Fonte: Autores.

Na Figura 2, destacam-se os artigos selecionados e os critérios de seleção e exclusão dos artigos para estudo. Após a pesquisa nos bancos de dados acadêmicos, o primeiro critério de exclusão, foi à pertinência ao tema e se o estudo abordava os pontos que queríamos observar, ou seja, estudo sobre a indústria 4.0 de fato. Após esse filtro, foi realizado um tratamento dos dados a partir dos artigos levantados, com o objetivo de identificar as áreas, subáreas da Engenharia de Produção que estão relacionadas com a Indústria 4.0, fazendo um comparativo com o que ABEPRO (2019a) define como parâmetros de ensino da engenharia de produção no Brasil. A ideia foi criar uma perspectiva de quais áreas e pontos estão sendo mais abordados, bem como quais tecnologias estão sendo difundidas no território nacional e de certa formar verificar a tendência da evolução do tema para os próximos anos. Além das áreas e subáreas, foram analisadas as palavras chaves mais citadas nas pesquisas, sendo possível ter uma visão panorâmica de quais os assuntos e temas mais difundidos e qual a sua evolução durante o período estudado.

Com um número expressivo e visando selecionar apenas trabalhos relevantes, foram levados em consideração as fontes de publicação e sua relevância, medido pela quantidade de citações, reduzindo a pesquisa inicial para 422 artigos. Uma última seleção foi necessária, pois muitos trabalhos fugiam do universo da Engenharia de Produção, deixando de contribuir com a análise desejada e causando certa distorção dos dados. Nessa etapa foram excluídos todos os artigos que não faziam parte da área de conhecimento da Engenharia de Produção. 


\section{Resultados e Discussão}

Em uma primeira pesquisa foram encontrados 5540 artigos, ao aplicarmos os critérios comentados na metodologia esse número foi reduzido para 181, a partir de então foi possível extrair informações sobre a evolução da indústria 4.0 no Brasil. A seguir, apresentam-se os principais resultados obtidos. A Figura 3 mostra a distribuição das publicações ao longo dos anos.

Figura 3. Quantidade de publicações por ano sobre o tema Indústria 4.0.

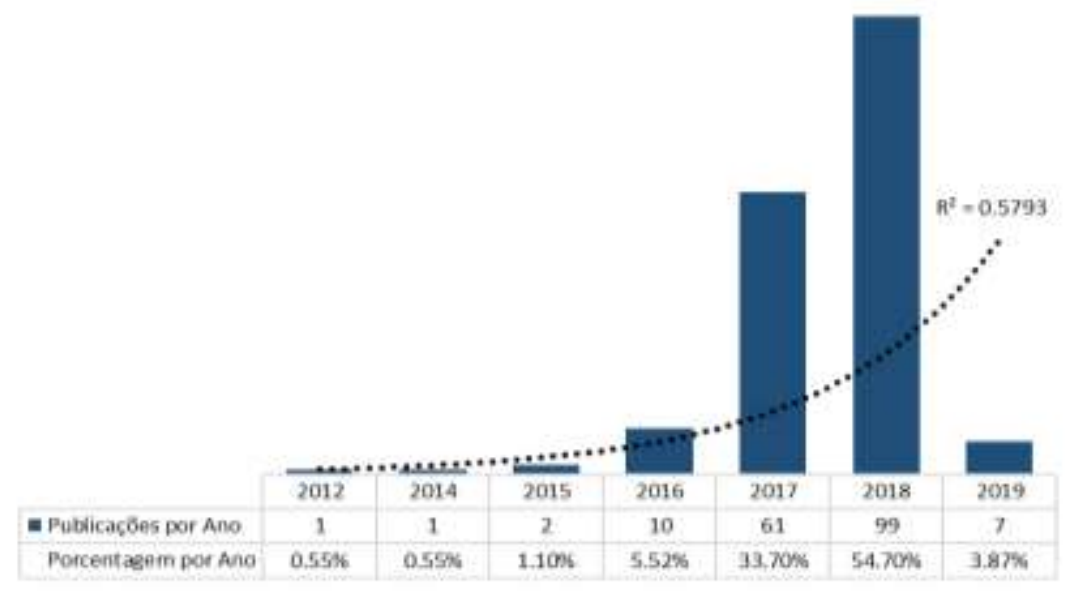

Fonte: Autores.

Na Figura 3, pode-se inferir que o tema indústria 4.0 começou a ser tratado no Brasil como uma solução na produção a partir de 2016. A partir desta data observa-se um crescimento exponencial, tendo em 2018, 99 publicações, totalizando mais da metade dos artigos já publicados no país. Vale destacar que as 7 publicações informadas nos anos de 2019, foram todas publicadas até o mês de março, quando as buscas foram finalizadas. Para se ter um parâmetro de comparação e possibilidade de realizarmos métricas nos dados obtidos, classificamos os mesmos de acordo com a divisão de áreas e subáreas que a Associação Brasileira de Engenharia de Produção (ABEPRO, 2019c) aplica. A Figura 4 exibe a distribuição dos artigos em relação a essas áreas da Engenharia de Produção.

Figura 4. Áreas relacionadas à Engenharia de Produção.

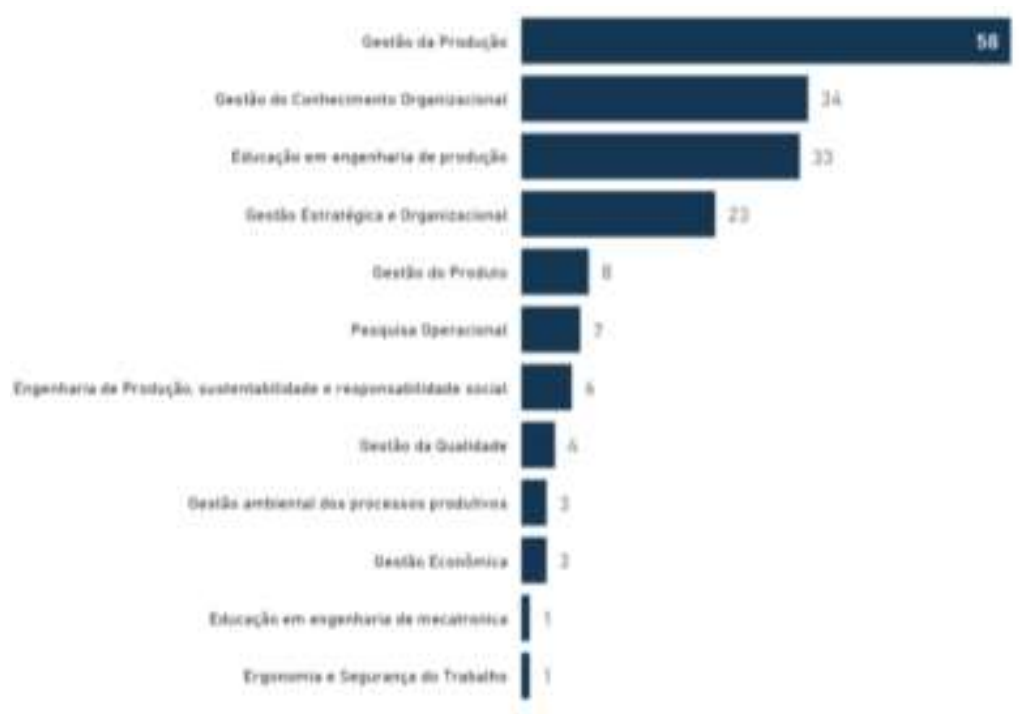

Fonte: Autores. 
Nota-se na Figura 4, que a área de Gestão de Produção é a que apresentou o maior número de trabalhos (58), seguida pela área de Gestão do Conhecimento Organizacional (34), Educação em Engenharia de Produção (33), e Gestão Estratégica Organizacional (23). Estas foram seguidas pelas demais áreas com menos de 10 trabalhos cada.

Para que fosse possível compreender quais são os assuntos mais discutidos dentro de cada área foi elaborada a Tabela 1, onde é possível ver a distribuição das publicações por subáreas. Percebe-se que a subárea com o maior número de publicações é a gestão de sistemas de produção, além de ser a subárea com o maior número de publicações no total essa também é a subárea mais publicada nos anos de 2017 e 2018 (Tabela 1). Curiosamente, enquanto estas pesquisas enfatizam a necessidade de modernização e adequação do setor industrial, outro estudo aponta evidências do fenômeno de desindustrialização, identificado no Brasil (Silva \& Nääs, 2020).

Tabela 1. Áreas e subáreas relacionadas a Engenharia de Produção.

\begin{tabular}{|c|c|c|c|c|c|c|c|c|}
\hline Area relacionada & 2012 & 2014 & 2015 & 2016 & 2017 & 2018 & 2019 & Total \\
\hline Gestía da Produçio & 1 & & 1 & 4 & 24 & 27 & 1 & $\$ 8$ \\
\hline Gestio de sistemas de produça & & & & 1 & 12 & 9 & & 22 \\
\hline Gestio de processos produtivon & & & 1 & 1 & 2 & 6 & 1 & 11 \\
\hline Logistica e gestào da cadeia de suprimertos & & & & 1 & 5 & s & & 10 \\
\hline Gestlo de operacbles e servicos & & & & & 6 & 3 & & 9 \\
\hline Gestio de manuterçĭo & & & & & & 2 & & 2 \\
\hline Gest3o de operacoer e produas. & 1 & & & & & & & 1 \\
\hline Placiejamentro o cretrele da produçio & & & & & & 1 & & 1 \\
\hline Projeto de Fibrica e de instalsçdes industriais & & & & & s. & & & 1 \\
\hline Semilacto da Produço & & & & 1 & & & & 1 \\
\hline Gestao do Conhecimento Organizacional & & & & 3 & 10 & 19 & 2 & 34 \\
\hline Gestälo de tecnelegin & & & & 2 & 5 & 7 & 1 & 15 \\
\hline Gest jo da novsţäo & & & & 1 & 5 & s & $i$ & 12 \\
\hline Entrategias de Produçalo & & & & & & 2 & & 2 \\
\hline Gestio da informaçás de produçaba e operaçbes & & & & & & 2 & & 2 \\
\hline Gestho do conhecimento em sotemas produtivo: & & & & & & 2 & & 2 \\
\hline Gastio de grojetos & & & & & & $i$ & & i \\
\hline Educasào em engenharia de produçáo & & & I & 2. & 14. & 15 & 1 & 33 \\
\hline Estudo do desenvolvimento e aplicaça da pesquisa em engerhana de produço & & & 1 & 1 & 7 & 7 & & 16 \\
\hline Eatudo do Entine de Engeritaris de Produçio & & & & 1 & 2 & 7 & 1 & 11 \\
\hline Ertudo da pratica profiasional am engenharia de produçąo & & & & & 5 & & & 5 \\
\hline Gestlo da teconologia & & & & & & 1 & & 1 \\
\hline Gestao Estrattegica \& Organizacional & & & & & 7 & 15 & 1 & 23 \\
\hline Plgnejemento estrategico e operadonal das estrutura organitacional & & & & & 3 & 11 & & 14 \\
\hline Estrategias de Produçä & & & & & & 1 & 1. & 2 \\
\hline Urganização induntrial & & & & & 1 & 1 & & 2 \\
\hline Gestio e Estrategia de mercado e Froduto & & & & & 1 & & & 1 \\
\hline Gestao e estratégis de mercados & & & & & & 1 & & I \\
\hline Gestalo e estratégra de mercados e produtos: & & & & & 1 & & & 1 \\
\hline Plariejzmento estrategico de regocio & & & & & & 1 & & 1 \\
\hline Redes de ensiesas e gest3o da cadeie produbva & & & & & 1 & & & 1 \\
\hline Gestio do Produto & & & & & 1. & 7 & & s \\
\hline Engenharia do Produto & & & & & 1 & 4 & & 5 \\
\hline Metodotogia se projeto do produto & & & & & & 2 & & 2 \\
\hline Planojaments do produto & & & & & & 1 & & 1 \\
\hline Pesquisa Operacional & & 1 & & & 1 & s & & 7 \\
\hline Modelagem analise e simulacto & & & & & & 3 & & 3 \\
\hline Cecisfo multicriterial & & 1 & & & & & & 1 \\
\hline Modelagem unblse e simulaça & & & & & & 1 & & 1 \\
\hline Drogramaça matemutica & & & & & & 1 & & 1 \\
\hline Teoria da decisajo e dos joges & & & & & 1 & & & 1 \\
\hline Engenharia de Produçio, sustentabilidade e responsabilidade social & & & & & 1 & 4 & 1 & 6 \\
\hline Responsabìicade social organizacional & & & & & & 2 & 1 & 3 \\
\hline Governança Orgarizacional & & & & & 1 & 2 & & 2 \\
\hline Gentäe da Qualidade & & & & & 1 & 3 & & 4 \\
\hline Qualidade en servicos & & & & & 1 & 2 & & 3 \\
\hline Engentharia económica & & & & & & 1 & & 1 \\
\hline Gestío ambiental dos processos produtivos & & & & 1 & 1 & 1 & & 3 \\
\hline Gestalo onergetica & & & & 1 & 1 & 1 & & 3 \\
\hline Gestäo Econòmica & & & & & 1 & 1 & 1 & 3 \\
\hline $\begin{array}{l}\text { Gestác de investimentos } \\
\text { Engenharia-económict }\end{array}$ & & & & & 1 & 1 & 1 & 1 \\
\hline Educą̧so en engenharia de mecatronica & & & & & & 1 & & 1 \\
\hline Ertude do ensino de engenharia de mecaitreniço & & & & & & 1 & & 1 \\
\hline Ergonomia e Segurança do Trabaitho & & & & & & 1 & & 1 \\
\hline Aralise e prevensato de riscos de acidentes & & & & & & 1 & & 1 \\
\hline Total & 1 & 1 & 2 & 10 & 61 & 99 & 7 & 141 \\
\hline
\end{tabular}

Fonte: Autores. 
Na Tabela 1 encontram-se os números das publicações nas áreas indicadas na Figura 4, com o desdobramento de suas subáreas, desde 2012 até 2019 e o total durante estes anos, totalizando 181 publicações no período estudado. É possível perceber também que as subáreas dos artigos publicados nos primeiros anos de pesquisa de 2012 a 2014, não receberam nenhuma publicação nos últimos anos pesquisados mostrando uma tendência de mudança no conceito de indústria 4.0 no Brasil.

A partir do levantamento das palavras chaves foi elaborada a Figura 5, para que fosse possível ver quais são as tecnologias mais comentadas quando o assunto é indústria 4.0 no Brasil.

Figura 5. Levantamento de tecnologias utilizadas.

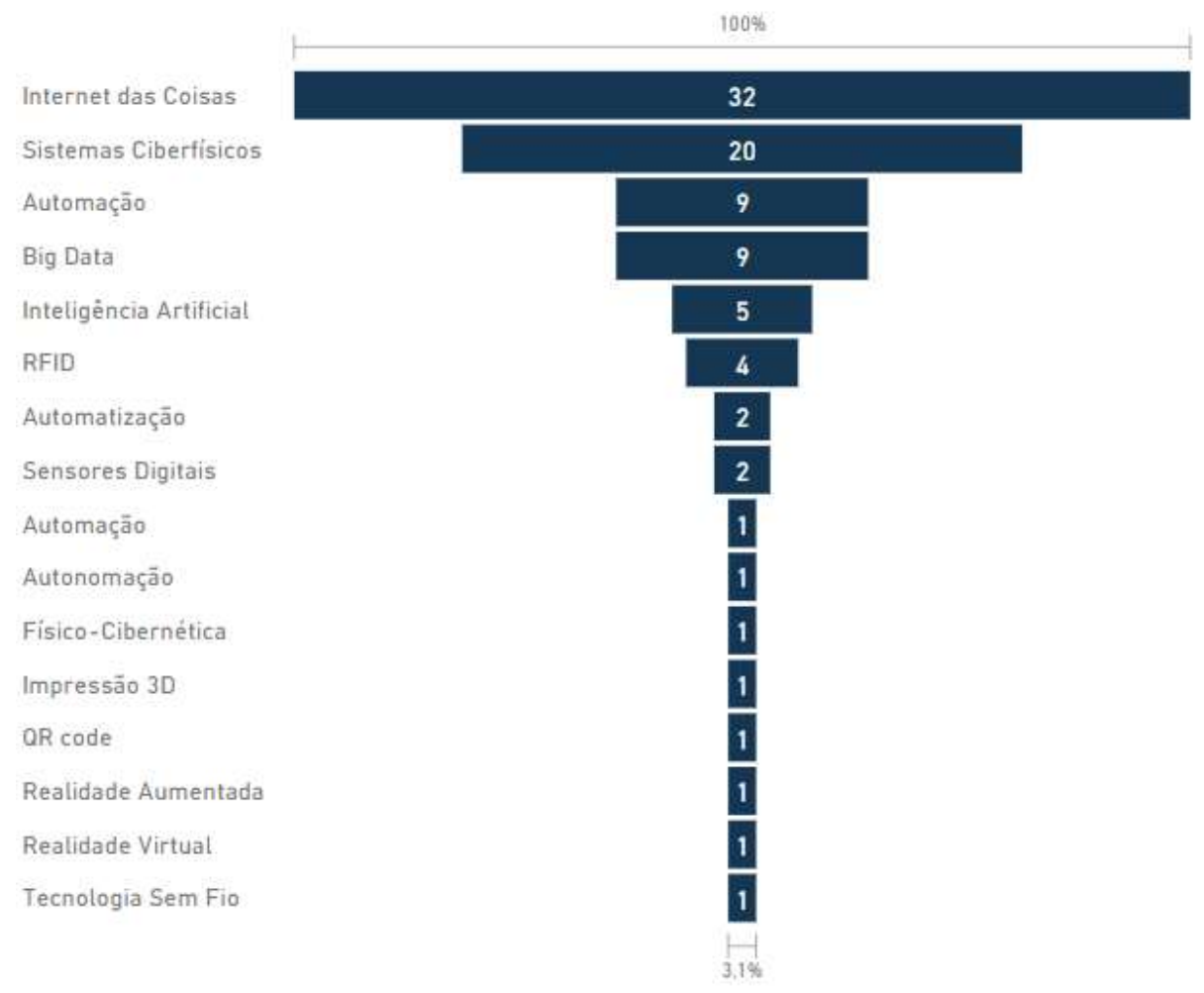

Fonte: Autores.

Pode-se notar na Figura 5, que a internet das coisas foi o termo que mais constou das palavras chaves (32\%), seguido pelo termo sistemas cibernéticos (20\%), sendo que as demais palavras-chaves tiveram menos que $10 \%$ das citações.

Com os dados obtidos, conseguiu-se traçar uma perspectiva de quais as habilidades serão exigidas de um Engenheiro de Produção, frente à essa nova revolução industrial. Pode-se inferir, que o perfil do Engenheiro 4.0 inclui: conhecimento sólido de disciplinas clássicas, denominadas como Hard Skills (mecânica, elétrica, eletrônica, informática, matemática, física); competências metodológicas sólidas baseadas em teoria de sistemas; conhecimento básico em informática e ciência de dados; conhecimento contextual em outras disciplinas, criatividade, pensamento analítico, lidar com complexidade e pressão, autonomia entre outros, classificadas como Soft Skills.

\section{Considerações Finais}

Os resultados indicam que o tema da indústria 4.0 está em crescimento exponencial dentro da comunidade científica no Brasil, alcançando um número expressivo de publicações a partir de 2016. Outros parâmetros que se pode notar na difusão do tema no país, são os investimentos com P\&D, importações e exportações líquidas de alta tecnologia, como indicada o índice 
Global de Inovações (GNI, 2018). Porém, quando comparado com os países pioneiros dessa revolução, o Brasil ainda está em estágio de descoberta e desenvolvimento do tema, fato evidenciado pela diferença de publicações e dados de investimento apresentado (MCTIC, 2018).

Para o perfil do Engenheiro de Produção 4.0, chegou-se à conclusão de que é preciso buscar uma combinação de educação adequada para a função a ser executada, que precisa ser adaptada, várias vezes, ao longo da carreira. Além dos conhecimentos clássicos, considerados como Hard Skills, fundamentos e conhecimentos de base das engenharias tradicionais, deve haver demanda crescente pela transformação, adaptabilidade, constante desenvolvimento e evolução perante o avanço da tecnologia e a obtenção competências Soft Skills. Pode-se também inferir que os temas mais pesquisados estão relacionados a Internet das coisas, sistemas ciber-físicos, automação e big data.

Recomenda-se, para futuros trabalhos, a continuidade da pesquisa com a atualização das publicações, de forma a se identificar a evolução do tema no Brasil.

\section{Referências}

ABDI (2019). Agência Brasileira de Desenvolvimento Industrial. Indústria 4.0 pode economizar R\$ 73 bilhões ao ano para o Brasil. HTTPS://WWW.ABDI.COM.BR/POSTAGEM/INDUSTRIA-4-0-PODE-ECONOMIZAR-R-73-BILHOES-AO-ANO-PARA-O-BRASIL.

ABEPRO (2002). Associação Brasileira de Engenharia de Produção. Diretrizes Curriculares para as Engenharias (CNE 11/03/2002). http://www.abepro.org.br/arquivos/websites/1/Res_CNE_11-03-2002.doc.

ABEPRO (2005). Associação Brasileira de Engenharia de Produção. Matriz de Conhecimento (Resolução 1010/05 CONFEA). http://www.abepro.org.br/arquivos/websites/1/Matriz\%20de\%20Conhecimento\%20-\%20CREA's.pdf.

ABEPRO (2019a). Associação Brasileira de Engenharia de Produção: Referências curriculares da Engenharia de Produção. http://www.abepro.org.br/arquivos/websites/1/Ref_curriculares_ABEPRO.pdf.

ABEPRO (2019b). Associação Brasileira de Engenharia de Produçã. Glossário técnico - área de Engenharia de Produção. http://www.abepro.org.br/interna.asp?ss=1\&c=585.

ABEPRO (2019c). Associação Brasileira de Engenharia de Produção. Tabela de áreas da Engenharia de Produção. http://www.abepro.org.br/arquivos/websites/1/\%C3\%81reas\%20da\%20Engenharia\%20de\%20Produ\%C3\%A7\%C3\%A3o.pdf.

Beyea, S. C., \& Nicoll, L. H. (1998). Writing an integrative review. AORN Journal, 67, 877-880.

Boligian, L., \&Alves, A. Geografia: espaço e vivência. Atual (2010). 151.

Botelho, L. L. R., de Almeida Cunha, C. C., \& Macedo, M. (2011). O método da revisão integrativa nos estudos organizacionais. Gestão e Sociedade, 5 (11), 121-136.

Broome, M. E. (2006). Integrative literature reviews for the development of concepts. In: Rodgers, B. L.; Castro, A. A. Revisão sistemática e meta-análise. https://www.metodologia.org/meta1.PDF.

CNI (2016). Confederação Nacional Da Indústria. Desafios para Indústria $4.0 \quad$ no Brasil. https://static.portaldaindustria.com.br/media/filer_public/d6/cb/d6cbfbba-4d7e-43a0-9784-86365061a366/desafios_para_industria_40_no_brasil.pdf.

Falcão, A. C. R. D. A. (2019). Sistematização dos Pilares da Indústria 4.0: uma análise utilizando revisão bibliográfica sistemática (Tese de doutorado, Universidade de São Paulo)

Firjan, 2016. Indústria 4.0. https://www.firjan.com.br/publicacoes/publicacoes-de-inovacao/industria-4-0-1.htm

GNI (2019). Global Innovation Index. Índice Global de Inovação https://static.portaldaindustria.com.br/portaldaindustria/n oticias/media/filer_public/01/ce/01ce693a-2a6c-43b9-a30a-2cdc3b0ce5b3/indice_global_de_inovacao_ingles.pdf.

Kagermann, H., Wahlster, W., \& Helbig, J. (2013). Securing the future of German manufacturing industry: Recommendations for implementing the strategic initiative Industrie 4.0. Final report of the Industrie 4.0.

Lee, J., Kao, H. A., \& Yang, S. (2014). Service innovation and smart analytics for industry 4.0 and big data environment. Procedia CIRP, 16, 3-8.

MCTIC (2018). Ministério da Ciência, Tecnologia, Inovações e Comunicações. Indicadores Nacionais de Ciência, Tecnologia e Inovação 2018. https://repositorio.mctic.gov.br/bitstream/mctic/3433/1/2018_indicadores_cti.pdf.

Pereira, A. S., et al. (2018). Metodologia da pesquisa científica. UFSM. https://repositorio.ufsm.br/bitstream/handle/1/15824/Lic_Computacao_MetodologiaPesquisa-Cientifica.pdf?sequence $=1$.

Sakurai, R., \& Zuchi, J. D. (2018). As revoluções industriais até a indústria 4.0. Revista Interface Tecnológica, 15(2), 480-491. 
Research, Society and Development, v. 10, n. 5, e10610513838, 2021

(CC BY 4.0) | ISSN 2525-3409 | DOI: http://dx.doi.org/10.33448/rsd-v10i5.13838

Santos, B. P., Silva, L. A., Celes, C. S. F. S., Borges, J. B., Neto, B. S. P., Vieira, M. A. M., \& Loureiro, A. (2016). Internet das coisas: da teoria à prática. Minicursos SBRC-Simpósio Brasileiro de Redes de Computadores e Sistemas Distribuídos, 31.

Siemens, A. (2017). Conceito de Indústria 4.0. Portugal: Siemens SA. Editado por Siemens AS. 23p.

Silva, A. V. da; Nääs, I. de A. (2020). Deindustrialization: A view of the Brazilian economy. Research, Society and Development, 9(12), e6591210494. 10.33448/rsd-v9i12.10494.

Sousa, F. R., Moreira, L. O., \& Machado, J. C. (2009). Computação em nuvem: Conceitos, tecnologias, aplicações e desafios. II Escola Regional de Computação Ceará, Maranhão e Piauí (ERCEMAPI), 150-175.

Sousa, R. (2019). Segunda Revolução Industrial. Brasil Escola. https://brasilescola.uol.com.br/historiag/segunda-revolucao-industrial.htm.

Taurion, C. (2013). Big data. Brasport. Technical Information.

Venturelli, M. (2016). Indústria 4.0: uma visão da automação industrial. Automação industrial. http://www. automacaoindustrial. info/industria-4-0-umavisao-da-automacaoindustrial.

Vermulm, R. (2018). Políticas para o Desenvolvimento da Indústria 4.0 No Brasil. IEDI (Organização). https://web.bndes.gov.br/bib/jspui/bitstream/1408/15486/1/POL\%c3\%8dTICAS\%20PARA\%20O\%20DESENVOLVIMENTO\%20DA\%20IND\%c3\%9aSTRI A\%204.0\%20NO\%20BRASIL_2018.pdf.

Whittemore, R., \& Knafl, K. (2005). The integrative review: Updated methodology. Journal of Advanced Nursing, 52(5), 546-553.

Wolf, D. F., Simões, E. V., Osório, F. S. \& Junior, O. T. (2009). Robótica móvel inteligente: Da simulação às aplicações no mundo real. In Mini-Curso: Jornada de Atualização em Informática (JAI), Congresso da SBC (p. 13). sn. 